\title{
Immune Mediated Necrotizing Myopathy: a Cause of Isolated Myopathy of Neck Extensor Muscle
}

\author{
Rahul Sehgal, MD; Rafael Medina-Flores, MD; Ralph Yachoui, MD; and Charles V Kenney, MD
}

Immune mediated necrotizing myopathy (IMNM) is a unique form of myositis that is characterized by distinct muscle biopsy features including abundant myofiber necrosis, degeneration, and regeneration with only minimal, if any, inflammation on muscle biopsy. IMNM is clinically similar to idiopathic inflammatory myopathy (IIM); hence, muscle biopsy is essential to diagnose IMNM. Herein we describe a case of neck extensor weakness due to necrotizing myopathy. Isolated weakness of the neck extensor muscles is uncommon in IIM and IMNM. This case describes the diagnostic work-up, treatments utilized, and 2 year follow-up course without involvement of other muscle groups and without progression of neck extensor muscle weakness. Advanced imaging using magnetic resonance imaging (MRI) facilitated the diagnosis by identifying the affected muscles and site for muscle biopsy.

Keywords: Myositis/diagnosis/therapy; Neck muscles/physiopathology; Neuromuscular diseases; Polymyositis/ physiopathology

I diopathic inflammatory myopathies (IIMs) are rare diseases with an insidious onset of muscle weakness. Primary idiopathic polymyositis (PM), primary idiopathic dermatomyositis (DM), juvenile dermatomyositis (JDM), inclusion body myositis (IBM), cancer-related myositis, and myositis with collagen vascular disease are included in the group of IIM disorders. Immune mediated necrotizing myopathy (IMNM) is a distinct form of myositis recognized in the diagnostic classification scheme in 2003. ${ }^{1}$ It is characterized by muscle fiber necrosis and phagocytosis and absent or only minimal inflammation on muscle biopsy. Of the several criteria published to date, the first set by Bohan and Peter $^{2,3}$ remain the mainstay of diagnosing IIM. Skeletal muscle involvement in IIM, except in IBM, is usually bilateral and symmetric in distribution. Patients with IBM have a predominantly distal or asymmetric involvement. Severity of the weakness and muscle group involvement are also different in IIM. With PM and DM, the weakness is worse in the proximal muscles and muscles of the lower extremities. ${ }^{4}$ Isolated involvement of the neck extensor muscles is rare in IIM and IMNM. Herein we present a middle-aged man with insidious onset of neck extensor weakness. This case outlines the broad differential diagnosis of neck extensor muscle weakness and highlights occurrence of neck extensor muscle weakness as the sole manifestation of IMNM.

A 60-year-old Caucasian man presented to the outpatient clinic with complaints of progressive head drop of an 8-month duration. He initially noted difficulty holding his head up spontaneously, but as time went on his symptoms worsened, requiring support with the hand in order to look up (figures 1 and 2). He reported diurnal variation with extensor fatiguing in the latter half of the day. He denied any history of neck pain/cervicalgia or headaches. He denied skeletal muscle weakness elsewhere in the body. There were no bowel or bladder problems, dysphagia, hoarseness, stridor, or sensory deficits. He reported no history of fever, shortness of breath, unexplained weight loss, or night sweats. He was up-to-date with age appropriate cancer screening. There was no family history of autoimmune muscle disease and no personal history of statin drug exposure. He did not smoke or drink alcohol. His medical history was notable for a stable pulmonary nodule, bilateral osteoarthritis of the knees, and mild psoriasis. He underwent multiple evaluations with various providers and chiropractors without a definitive diagnosis.

Physical examination was remarkable for reversal of normal cervical lordosis curvature. Cervical range of motion was limited to 25 degrees extension, 10-15 degrees lateral flexion, and 30 degrees lateral rotation bilaterally. Neurological examination revealed atrophy of neck extensors and trapezius muscles and weakness of the neck extensor (3/5), while neck flexor strength was intact (5/5). Sensory examination and deep tendon reflexes were normal. No features of Raynaud's phenomenon, skin rash, inflammatory arthritis, or sclerodactyly were apparent. 

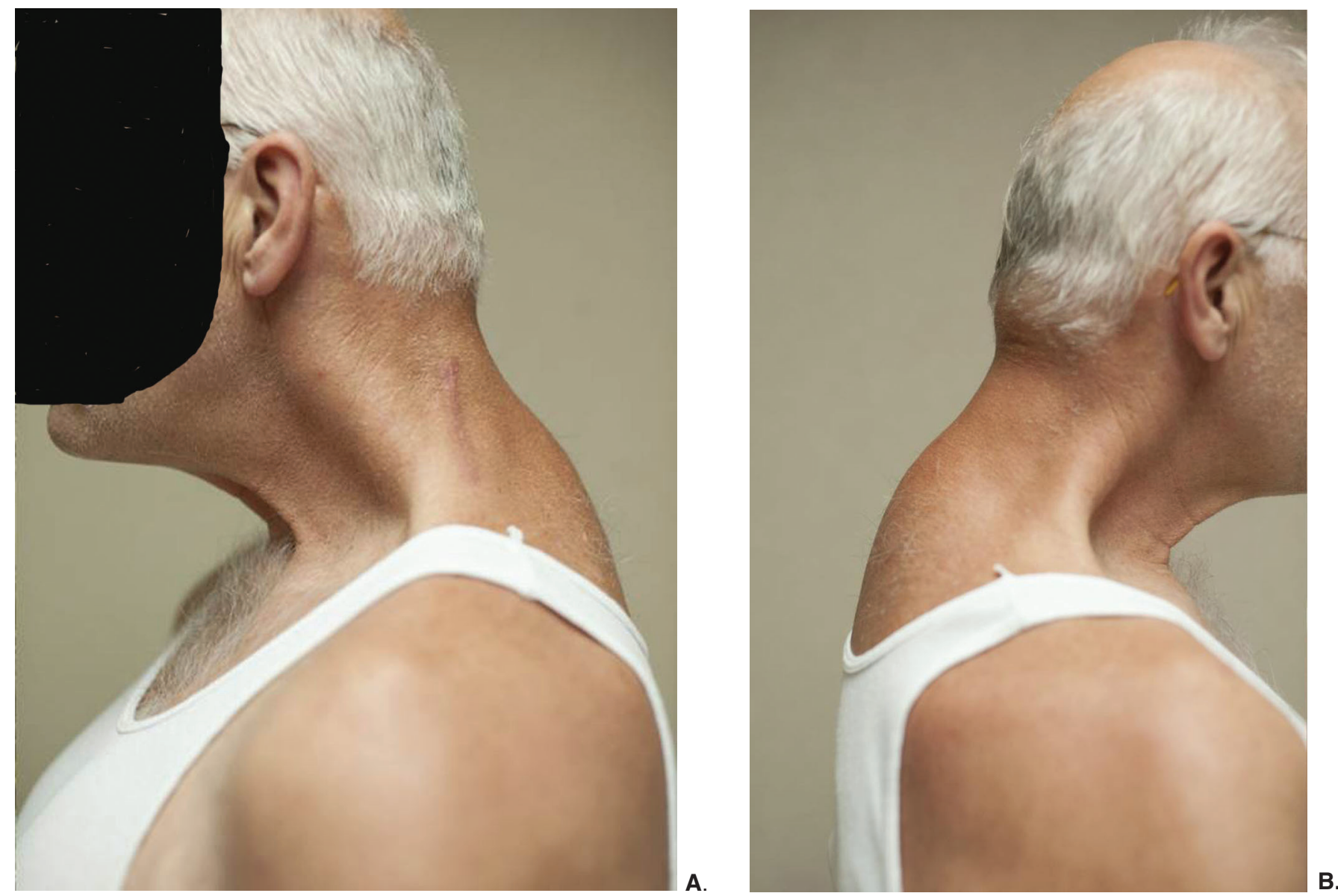

Figure 1. Photographs of patient that demonstrate severe weakness and wasting of neck extensor muscles. Scar mark (A) shows site of muscle biopsy from left trapezius muscle.

Laboratory evaluation was notable for elevated creatine kinase (CK) at $568 \mathrm{U} / \mathrm{L}$ (range 50-235 U/L), aldolase at 8.9 $\mathrm{U} / \mathrm{L}$ (range 2.3-7.1 U/L), and lactate dehydrogenase (LDH) at $211 \mathrm{U} / \mathrm{L}$ (range 80-190 U/L). Complete blood count, renal and hepatic function, and electrolytes were all normal. Antinuclear antibody was negative. Tests for human leukocyte antigen (HLA)-B27, rheumatoid factor, and acetylcholine receptor binding antibody were absent. Tests for hepatitis B, $\mathrm{C}, \mathrm{HIV}$, and tick borne illnesses were negative as well.

Magnetic resonance imaging (MRI) of the cervical spine showed multilevel cervical spondylosis with broad-based disc bulging from $\mathrm{C} 3$ to $\mathrm{C} 7$ with facet hypertrophy and mild encroachment upon the ventral cerebral spinal fluid space at C5 to C6 level without any evidence of intramedullary signal change within the cord. MRI of the neck musculature with axial T1 and short tau inversion recovery (STIR) sequences revealed patchy areas of edema with areas of fatty infiltration, compatible with myositis of the posterior para-spinous musculature, sparing the levator scapulae and right trapezius (figure 3).

Electromyogram and nerve conduction velocity testing revealed localized myopathy with increased insertional activity in bilateral lower cervical (C6-C7) paraspinal muscles. There was no evidence of diffuse denervation such as would occur with amyotrophic lateral sclerosis. Ulnar, median, radial, and sural sensory studies were normal. Motor unit potentials at the level of the upper cervical, lower lumbar paraspinal muscles, biceps brachii, triceps brachii, tibialis anterior, and gastrocnemius muscles were normal.

Muscle biopsy from the left trapezius muscle revealed chronic active necrotizing myopathy (figure 3) with endomysial mononuclear inflammatory infiltrate without perifascicular atrophy. Major histocompatibility complex class 1 (MHC-1) immunostain showed strong and diffuse membranous reactivity in all myofibers as well as deposition of complement on capillaries and non-necrotic muscle fibers. No features of metabolic, mitochondrial, or nemaline myopathy were seen.

An immune-mediated necrotizing myopathy was favored as a diagnosis, based on the distinct histopathologic features. Clinical examination and electrodiagnostic studies favored isolated involvement of the neck extensor muscles. Myositis antibody profile was negative for myositis specific (Jo-1, Mi-2, PL-7, PL-12, EJ, OJ, SRP) and myositis associated (PM-Scl, U1RNP, U2RNP, Ro) antibodies.

The patient was treated with $80 \mathrm{mg}$ of prednisone a day $(\sim 1 \mathrm{mg} / \mathrm{kg})$, which was gradually weaned off over next 3-month period. Methotrexate was started with high dose 

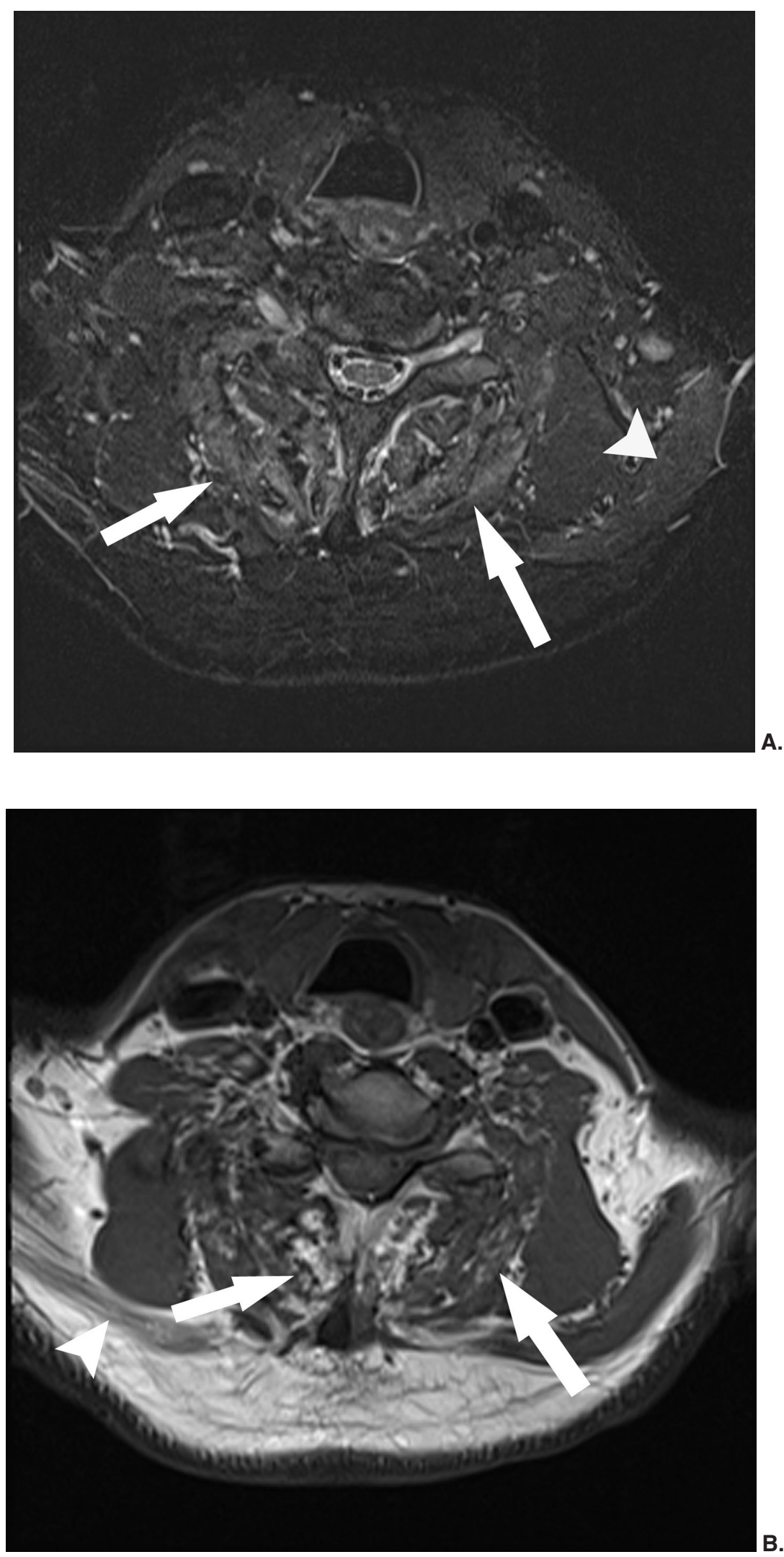

Figure 2. Magnetic resonance imaging of the neck paraspinal musculature: (A) The paraspinous musculature shows abnormal signal intensity on the STIR image, compatible with areas of bilateral active muscle edema, suggestive of myositis (arrows). There is also mild involvement at the left trapezius (arrowhead). (B) On the accompanying T1-weighted images, there is high signal fatty infiltration consistent with mild fatty infiltration (arrows.) The right trapezius shows severe atrophy (arrowhead). 


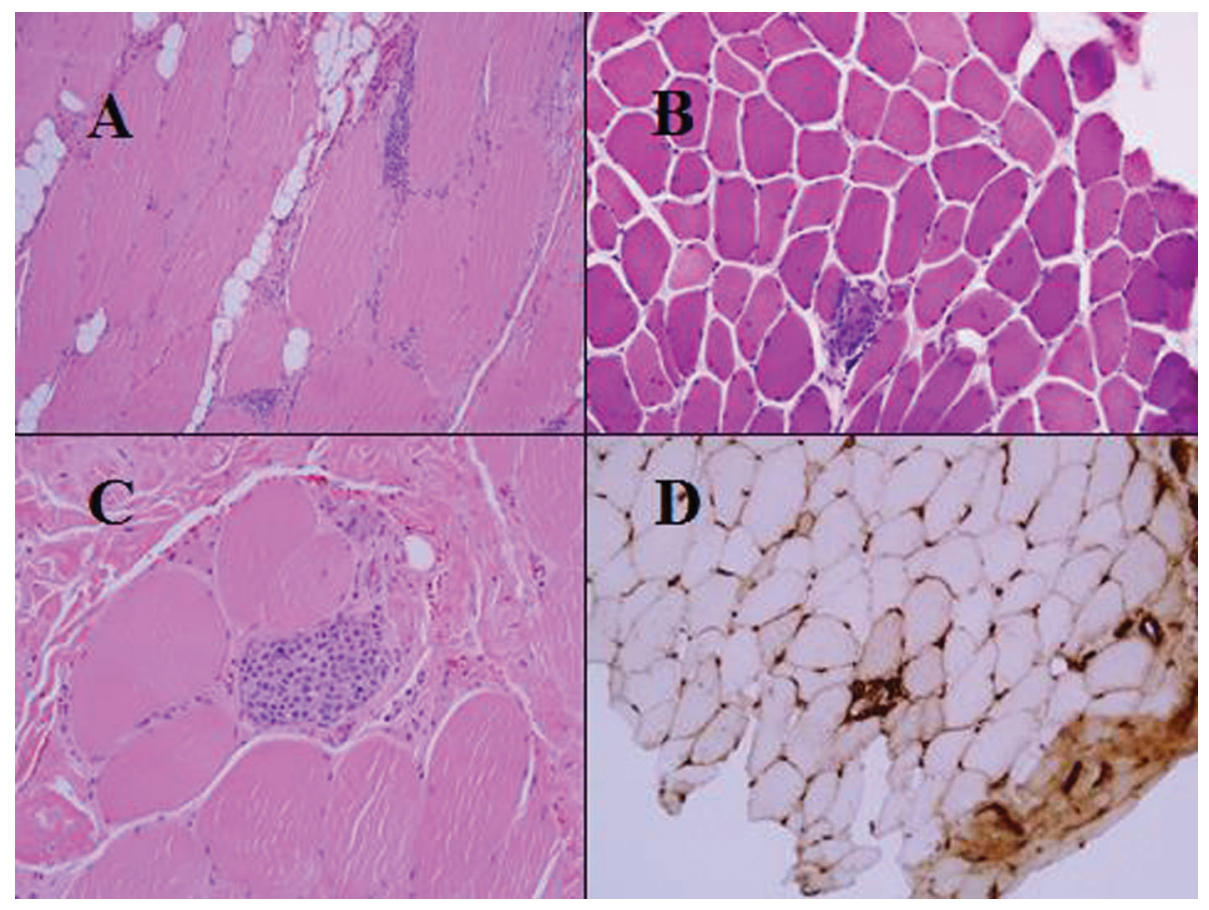

Figure 3. (A) On formalin-fixed paraffin embedded muscle sections there were endomysial mononuclear infiltrates, and mild fatty infiltration. Hematoxylin and eosin, 10x. (B) On frozen section, there were frequent angulated atrophic fibers and scattered degenerating fibers. Hematoxylin and eosin, 40x. (C) On formalin-fixed paraffin embedded muscle sections there were frequent areas of muscle regeneration. Hematoxylin and eosin, 40x. (D) Degenerating fibers show strong immunoreactivity with major histocompatibility complex-1, 40x.

prednisone at a dose of $15 \mathrm{mg}$ per week. He took methotrexate for 3 months, which he subsequently discontinued due to lack of response. The patient thereafter declined further treatment with any other immunomodulatory drugs. His muscle enzymes remained persistently elevated without further decline in neck extensor muscle strength. No improvement to neck extensor muscle strength ensued. He did not develop further weakness in muscle strength of the upper or lower extremity muscles during 2 years of follow-up.

\section{Discussion}

Isolated weakness of the neck extensor muscles causing dropped head syndrome is a rare though disabling presentation of various neuromuscular diseases. The term 'dropped head syndrome' was first described by Suarez and Kelly ${ }^{5}$ as a noninflammatory, non-progressive condition with a benign course that is non-responsive to treatment with corticosteroids. Katz et $\mathrm{al}^{6}$ later coined the term 'isolated neck extensor myositis' (INEM) in their description of four patients with neck extensor muscle weakness. The differential diagnosis of neuromuscular disorders with prominent neck extensor weakness is broad (Table 1). Myositis of the neck flexors in IIM is common, although weakness of the neck extensors resulting in dropped or floppy head is very rare. ${ }^{7}$ Individual case reports of dropped head have been described in Parkinson's disease ${ }^{8}$ and after hemispheric striatal infarction. ${ }^{9}$ Nine cases of dropped head syndrome associated with systemic autoimmune diseases have also been described. ${ }^{10}$
Immune mediated necrotizing myopathy (IMNM), recognized by necrosis with mild or no inflammation, occurs in various conditions such as dermatomyositis, antisynthetase syndrome, statin use, anti-signal recognition particle-associated myopathy, or scleroderma associated myositis. ${ }^{11}$ The clinical presentation of IMNM is similar to IIM; hence, muscle biopsy is necessary for diagnosing IMNM.

Therapies utilized in INEM based on case reports include systemic steroids, intravenous immunoglobulins, and other immunosuppressants such as methotrexate, mycophenolate mofetil, and azathioprine. ${ }^{12,13}$ Treatment response to the various strategies has been variable. Our patient was started on supervised physical therapy for strengthening of the affected neck muscles, and he was treated with highdose glucocorticoids and methotrexate followed by azathioprine, with no improvement in neck extensor muscle weakness. The neck extensor weakness remained stable after an initial several months of decline with mild elevation of muscle enzymes. He remains employed fulltime, and he did not develop skeletal muscle weakness elsewhere, even after 2.5 years of follow-up. To our knowledge there is only one other report of a case of biopsy-positive necrotizing myopathy with involvement of the neck extensor muscles. ${ }^{14}$ That patient, however, also 
had muscle weakness in the proximal muscles of the upper and lower extremities. Our case is unique in that motor weakness remained confined to the neck extensor muscles without clinical signs of relapse in the follow-up period of 2.5 years.

INEM constitutes an isolated myopathy of neck extensor muscles. It is distinct from focal myositis, a condition of skeletal muscle characterized by focal enlargement within a single muscle and histopathologic features of inflammatory myopathy. ${ }^{15}$ The latter generally has a favorable prognosis and does not require sustained immunosuppressive therapy. The etiology of INEM is unknown. A monophasic focal inflammatory process restricted to neck extensor muscles has been postulated. ${ }^{16}$ A mechanical stretch from kyphotic posture and age-related loss of tissue elasticity producing injury to

Table 1. Neuromuscular causes and salient features of prominent neck extensor weakness.

Isolated neck extensor myositis (INEM)

Myasthenia gravis (MG)

Amyotrophic lateral sclerosis (ALS)

Polymyositis (PM)

Dermatomyositis (DM)

Inclusion body myositis (IBM)

Carnitine deficiency

Facioscapulohumeral dystrophy

Chronic inflammatory demyelinating polyneuropathy (CIDP)

Myotonic dystrophy

Endocrine disorders (hypothyroidism, hyperparathyroidism)

Parkinson's disease (PD)

Acute ischemic stroke

Scleromyositis

Congenital myopathy

EMG, electromyelogram; CK , creatine kinase; MRI, magnetic resonance imaging months to PM demyelination on nerve biopsy. modestly elevated CK neurologic deficits. mechanic' hands.
Benign, non-progressive clinical course, unresponsive to corticosteroid therapy. No inflammation on muscle biopsy, normal muscle enzymes and myopathic EMG. No other muscle groups involved

Limb girdle, facial weakness and ptosis. Acetylcholine receptor antibody (AchR Ab) presence. Absence of MRI abnormalities.

Severe neck (usually flexor muscle) and trunk weakness. Tongue fasciculations, elevated CK and diffuse denervation on EMG. Presence of upper and lower motor neuron signs.

Marked elevation of CK, symmetric proximal limb weakness, myopathic changes on EMG. Progression to generalized muscle weakness over 3-6

Prominent skin manifestations including Gottron's papules, heliotrope rash on eyelids and photosensitive skin rash. Skin rash may precede, follow or develop simultaneously with symptoms of myopathy. Other features similar

Slowly progressive clinical course, development of severe muscle atrophy and unresponsive to corticosteroid treatment. Distal muscle involvement causing finger flexor weakness and frequent falls due to quadriceps atrophy usually present in IBM patients

Symmetric involvement of upper/lower extremities and involvement of proximal and distal muscles. Weakness more than sensory symptoms and elevated cerebrospinal fluid protein without pleocytosis. Reduced deep tendon reflexes, weakness more than sensory symptoms, demyelinating neuropathy on nerve conduction velocity and evidence of segmental

Scapular winging, waddling gait, ptosis and proximal limb weakness. Weakness of neck extensors and flexors.

Partial deletion of the D4Z4 repeats near chromosome $4 q$ telomere at $4 q 35$. Insidious onset of facial muscle weakness initially progressing to pelvic girdle weakness. Myopathic EMG, presence of perivascular, endomysial and perimysial inflammation on muscle biopsy. Normal or

Myotonic potentials on EMG, elevated CK and facial weakness.

Abnormal thyroid stimulating hormone or parathyroid hormone levels. Responsive to treatment of underlying endocrine disorder.

Bradykinesia, rigidity and resting tremors present. Incidence of dropped head in PD is less than 5-6\%. Responsive to treatment with dopamine agonists or muscle afferent block of sternocleidomastoid. ${ }^{8}$

One case report. ${ }^{9}$ Abnormal MRI brain and presence of other focal

Two case reports. ${ }^{10,16}$ Overlapping syndrome of scleroderma, Raynaud's disease, arthralgia/arthritis, myalgia/myositis, interstitial lung disease,

Onset in infancy or childhood. Hypotonia and weakness (proximally>distally). Management is supportive 
cervical paraspinal muscles has also been proposed by Katz and colleagues. ${ }^{6}$ Treatment response in INEM is variable. In a case series, four out of the five patients had a relatively benign course, while one patient had further progression of weakness and involvement of neck flexors and proximal muscles. ${ }^{6}$ This is unlike IIM, which may begin as a focal disorder with secondary generalization varying in severity from mild disease to extreme disability.

\section{Conclusion}

Isolated weakness of the neck extensor muscles is a rare condition. A thorough investigation for neuromuscular and systemic autoimmune diseases should be performed in all cases of isolated neck extensor weakness. The treatment decision is challenging, due to the rarity of the illness, and treatment responses are variable. Physical therapy should, however, be part of any treatment strategy. The prognosis is usually favorable, though involvement of other muscle groups may rarely occur.

\section{References}

1. Hoogendijk JE, Amato AA, Lecky BR, Choy EH, Lundbert IE, Rose MR, Vencovsky J, de Visser M, Hughes RA. 119th ENMC international workshop: trial design in adult idiopathic inflammatory myopathies, with the exception of inclusion body myositis, 1012 October 2003. Naarden, The Netherlands. Neuromuscul Disord 2004;14:337-345.

2. Bohan A, Peter JB. Polymyositis and dermatomyositis (first of two parts). N Engl J Med 1975;292:344-347.

3. Bohan A, Peter JB. Polymyositis and dermatomyositis (second of two parts). N Engl J Med 1975;292:403-407.

4. Harris-Love MO, Shrader JA, Koziol D, Pahlajani N, Jain M, Smith M, Cintas HL, McGarvey CL, James-Newton L, Pokrovnichka A, Moini B, Cabalar I, Lovell DJ, Wesley R, Plotz PH, Miller FW, Hicks JE, Rider LG. Distribution and severity of weakness among patients with polymyositis, dermatomyositis and juvenile dermatomyositis. Rheumatology (Oxford) 2009;48:134-139.

5. Suarez GA, Kelly JJ. The dropped head syndrome. Neurology 1992;42:1625-1627.

6. Katz JS, Wolfe GI, Burns DK, Bryan WW, Fleckenstein JL, Barohn RJ. Isolated neck extensor myopathy: a common cause of dropped head syndrome. Neurology 1996:46:917921.

7. Mastaglia FL, Garlepp MJ, Phillips BA, Zilko PJ. Inflammatory myopathies: clinical, diagnostic and therapeutic aspects. Muscle Nerve 2003:27:407-425.

8. Fujimoto K. Dropped head in Parkinson's disease. J Neurol 2006;253:21-26.

9. Funabe S, Ryota T, Hayashi A, Yamashiro K, Shimura H, Hattori N. Reversible dropped head syndrome after hemispheric striatal infarction. J Stroke Cerebrovasc Dis 2014;23:785 787

10. Fernandez-Serena M, Arboleya L, Alonso S, Queiro R, Alperi M. Dropped head syndrome in a patient with scleromyositis. J Clin Rheumatol 2013;19:32-34.

11. Mammen A. Necrotizing myopathies: beyond statins. Curr Opin Rheumatol. 2014;26:679 683.

12. Muppidi S, Saperstein D, Shaibani A, Nations P, Vernito S, Wolfe G. Isolated neck extensor myopathy: is it responsive to immunotherapy? J Clin Neuromuscul Dis 2010;12:26-29.

13. Larsen H, Bogaard P, Oppel L. A case of isolated neck extensor myopathy responding favorably to immunotherapy. J Clin Neuromuscul Dis 2013;15:73-76.
14. Goh KJ, Wong KT, Tan CT. Myopathic dropped head syndrome: a syndrome of mixed aetiology. J Clin Neuromuscul Dis 2000;7:334-336.

15. Smith AG, Urbanits S, Blaivas M, Grisold W, Russell JW. Clinical and pathologic features of focal myositis. Muscle Nerve 2000;23:1569-1575.

16. Garcin B, Lenglet T, Dubourg O, Mesnage V, Levy R. Dropped head syndrome as a presenting sign of scleromyositis. J Neurol Sci 2010;292:101-103.

\section{Author Affiliations}

Rahul Sehgal, MD*; Rafael Medina-Flores, MD†; Ralph

Yachoui, MD*; Charles Kenney, MD

*Department of Rheumatology, Marshfield Clinic, Marshfield, Wisconsin, USA

†Department of Lab/Pathology, Marshfield Clinic, Marshfield, Wisconsin, USA

tDepartment of Radiology, Marshfield Clinic, Marshfield, Wisconsin, USA 